Pacific Journal of Mathematics

ASYMPTOTIC EXPANSIONS OF THE LEBESGUE CONSTANTS
FOR JACOBI SERIES 


\title{
ASYMPTOTIC EXPANSIONS OF THE LEBESGUE CONSTANTS FOR JACOBI SERIES
}

\section{L. FRENZEN AND R. Wong}

\begin{abstract}
Explicit expressions are obtained for the implied constants in the two $O$-terms in Lorch's asymptotic expansions of the Lebesgue constants associated with Jacobi series [Amer. J. Math., 81 (1959), 875-888]. In particular, a question of Szegö concerning asymptotic monotonicity of the Lebesgue constants for Laplace series is answered. Our method differs from that of Lorch, and makes use of some recently obtained uniform asymptotic expansions for the Jacobi polynomials and their zeros.
\end{abstract}

1. Introduction and summary. The $n$th partial sum of the Fourier series of an arbitrary function can be written in the form of an integral involving the Dirichlet kernel. The integral of the absolute value of this kernel is known as the $n$th Lebesgue constant, and is usually denoted by

$$
L_{n}=\frac{1}{\pi} \int_{0}^{\pi} \frac{\left|\sin \left(n+\frac{1}{2}\right) t\right|}{\sin (t / 2)} d t
$$

see $\left[19\right.$, p. 172]. The behavior of the sequence $\left\{L_{n}\right\}$ is closely connected with convergence and divergence properties of Fourier series, and the importance of this sequence has led many mathematicians to be concerned not only with just its asymptotic formula but also with its full asymptotic expansion. First, Fejer [1] showed that

$$
L_{n}=\frac{4}{\pi^{2}} \log n+c_{0}+\frac{c_{1}}{n}+\frac{\alpha(n)}{n^{2}},
$$

where $c_{0}$ and $c_{1}$ are constants and $\alpha(n)=O(1)$ as $n \rightarrow \infty$. An explicit expression was given for $c_{0}$ but not for $c_{1}$. Later, infinite asymptotic expansions were derived by Gronwall [4], Watson [18] and Hardy [7].

In an entirely analogous manner, the $n$th partial sum of the expansion of an arbitrary function in terms of Jacobi polynomials can be written as an integral involving a kernel; see, e.g., $[17$, p. 39]. The $n$th Lebesgue constant in this case has the integral representation

$$
\begin{aligned}
L_{n}(\alpha, \beta)= & \frac{\Gamma(n+\alpha+\beta+2)}{\Gamma(\alpha+1) \Gamma(n+\beta+1)} \\
& \cdot \int_{0}^{\pi}\left(\sin \frac{\theta}{2}\right)^{2 \alpha+1}\left(\cos \frac{\theta}{2}\right)^{2 \beta+1}\left|P_{n}^{(\alpha+1, \beta)}(\cos \theta)\right| d \theta .
\end{aligned}
$$


This result is due to Rau [12]. In view of the identity [17, p. 60]

$$
P_{n}^{(1 / 2,-1 / 2)}(\cos \theta)=\frac{\Gamma\left(n+\frac{3}{2}\right)}{\Gamma(n+1) \Gamma\left(\frac{3}{2}\right)} \frac{\sin \left(n+\frac{1}{2}\right) \theta}{(2 n+1) \sin (\theta / 2)},
$$

Equation (1.3) gives $L_{n}\left(-\frac{1}{2},-\frac{1}{2}\right)=L_{n}$, contrary to a statement made in [8, footnote 7].

Rau [12] was the first to show that for $\alpha>-1 / 2$ and $\beta>-1$,

$$
L_{n}(\alpha, \beta)=A_{\alpha \beta} n^{\alpha+1 / 2}+o\left(n^{\alpha+1 / 2}\right), \quad n \rightarrow \infty,
$$

where

$$
A_{\alpha \beta}=\frac{2}{\pi^{3 / 2}} \frac{\Gamma(\alpha / 2+1 / 4) \Gamma(\beta / 2+3 / 4)}{\Gamma(\alpha+1) \Gamma([\alpha+\beta] / 2+1)} .
$$

Later Szegö [16] had an alternative proof of (1.5), and furthermore showed that

$$
L_{n}\left(-\frac{1}{2}, \beta\right)=\frac{4}{\pi^{2}} \log n+o(\log n)
$$

for $\beta>-1$, and that for $-1<\alpha<-\frac{1}{2}$ and $\beta>-1$

$$
L_{n}(\alpha, \beta)=\frac{2^{-\alpha}}{\Gamma(\alpha+1)} \int_{0}^{\infty} \theta^{\alpha}\left|J_{\alpha+1}(\theta)\right| d \theta+o(1),
$$

where $J_{\alpha+1}(\theta)$ is the Bessel function of first kind.

The above results have been sharpened by Lorch $[8,9]$, particularly in the cases $\alpha=-\frac{1}{2}$ and $-\frac{1}{2}<\alpha<\frac{1}{2}$. For $-\frac{1}{2}<\alpha<<\frac{1}{2}, \alpha-\beta<1$ and $\beta>-1$, Lorch's result can be stated as follows:

$$
L_{n}(\alpha, \beta)=A_{\alpha \beta} n^{\alpha+1 / 2}+B_{\alpha}+O\left(n^{\alpha-1 / 2}\right)+O\left(n^{\alpha-\beta-1}\right)
$$

where

$$
\begin{aligned}
B_{\alpha}=\frac{2^{-\alpha}}{\Gamma(\alpha+1)}\{ & -M_{1}(\alpha)+\int_{0}^{j_{1}} x^{\alpha} J_{\alpha+1}(x) d x \\
& +2 \alpha \sum_{k=1}^{\infty}(-1)^{k} \int_{j_{k}}^{j_{k+1}} x^{\alpha-1} J_{\alpha}(x) d x \\
& \left.+2 \sum_{k=1}^{\infty}\left[M_{k}(\alpha)-\frac{\sqrt{2}}{\pi^{3 / 2}} \int_{j_{k-1}}^{j_{k}} x^{\alpha-1 / 2} d x\right]\right\}
\end{aligned}
$$

both infinite series being absolutely convergent. (Equation (4) in [9] contains two misprints; $M_{k+1}(\alpha)$ should be replaced by $M_{k}(\alpha)$ and $M_{1}(\alpha)$ should have a minus sign.) In (1.10), $j_{n}=j_{\alpha+1, n}$ is the $n$th positive zero of 
$J_{\alpha+1}(x), n=1,2, \ldots, j_{0}=0$, and

(1.11) $M_{k}(\alpha) \equiv(-1)^{k}\left(j_{\alpha+1, k}\right)^{\alpha} J_{\alpha}\left(j_{\alpha+1, k}\right)>0, \quad k=1,2, \ldots$

For $\alpha=-\frac{1}{2}$ and $\beta>-1$, Lorch also obtained the result that as $n \rightarrow \infty$,

$$
\begin{aligned}
L_{n}\left(-\frac{1}{2}, \beta\right)= & \frac{4}{\pi^{2}} \log n+C_{\beta} \\
& +O\left(n^{-1} \log n\right)+O\left(n^{-\beta-3 / 2}\right),
\end{aligned}
$$

where

$$
\begin{aligned}
C_{\beta}= & \frac{8}{\pi^{2}} \log 2+\frac{2}{\pi} \int_{0}^{1} \theta^{-1} \sin \theta d \theta \\
& -\frac{4}{\pi^{2}} \int_{0}^{\pi / 2} \frac{1-(\cos \theta)^{\beta+1 / 2}}{\sin \theta} d \theta \\
& -\frac{2}{\pi} \int_{1}^{\infty} \theta^{-1}\left\{\frac{2}{\pi}-|\sin \theta|\right\} d \theta
\end{aligned}
$$

the last integral being convergent. (There is a typographical error in [9, (8)]; the factor in front of $\log 2$ should be $8 / \pi^{2}$ and not $4 / \pi^{2}$.)

Lorch's investigation [10] was motivated by a question raised by Szegö concerning asymptotic monotonicity of the sequence $\left\{L_{n}(0,0)\right\}$; see also the editor's comment at the end of [13]. The result in (1.9), however, fails to answer the question of Szegö. Lorch thus posed to us in 1980 the problem of replacing the $O$-terms in (1.9) and (1.12) by explicitly determined expressions plus terms of lower asymptotic order. The following results provide a solution to his problem, and were announced in [3]. The detailed proofs of these results are the contents of the present paper. The fact that $\left\{L_{n}(0,0)\right\}$ is an asymptotically increasing sequence is an immediate consequence of the result given in (1.18) below.

First, for the restricted range $-\frac{1}{2}<\alpha<\frac{1}{2}$ and $-\frac{1}{2}<\beta<\frac{1}{2}$, we have

$$
\begin{aligned}
L_{n}(\alpha, \beta)= & A_{\alpha \beta} n^{\alpha+1 / 2}+B_{\alpha}+C_{\alpha \beta} A_{\alpha \beta} n^{\alpha-1 / 2} \\
& +\frac{1}{\Gamma(\alpha+1)} D_{\beta} n^{\alpha-\beta-1}+O\left(n^{\alpha-3 / 2}\right),
\end{aligned}
$$

where

$$
C_{\alpha \beta}=\frac{(\alpha+\beta+2)(\alpha+1 / 2)}{2}
$$


and

$$
\begin{aligned}
D_{\beta}=2^{-\beta} \sum_{k=1}^{\infty}\left[\hat{M}_{k}(\beta)\right. & -\frac{\sqrt{2}}{\pi^{3 / 2}} \int_{J_{k-1}^{\prime}}^{j_{k}^{\prime}} x^{\beta+1 / 2} d x \\
& \left.-\frac{\beta+1 / 2}{\sqrt{2 \pi}} \int_{j_{k-1}^{\prime}}^{j_{k}^{\prime}} x^{\beta-1 / 2} d x\right] .
\end{aligned}
$$

In (1.16), $j_{n}^{\prime} \equiv j_{\beta, n}$ is the $n$th positive zero of $J_{\beta}(x), n=1,2, \ldots, j_{0}^{\prime}=0$ and

$$
\begin{aligned}
\hat{M}_{k}(\beta) & =(-1)^{k}\left(j_{\beta, k}\right)^{1+\beta} J_{\beta-1}\left(j_{\beta, k}\right) \\
& =(-1)^{k+1}\left(j_{\beta, k}\right)^{1+\beta} J_{\beta+1}\left(j_{\beta, k}\right) .
\end{aligned}
$$

In the important particular case of Laplace series (i.e., the series in terms of Legendre polynomials at the end point $x=1$ ), $\alpha=\beta=0$, and (1.14) becomes

$$
\begin{aligned}
L_{n}(0,0)= & \frac{2^{3 / 2}}{\sqrt{\pi}} n^{1 / 2} \\
& +\left[1+2 \sum_{k=1}^{\infty}\left\{M_{k}(0)-\frac{2^{3 / 2}}{\pi}\left[k^{1 / 2}-(k-1)^{1 / 2}\right]\right\}\right] \\
& +\sqrt{\frac{2}{\pi}} n^{-1 / 2}+n^{-1} \sum_{k=1}^{\infty}\left\{\hat{M}_{k}(0)-\frac{2^{3 / 2}}{3}\left[k^{3 / 2}-(k-1)^{3 / 2}\right]\right. \\
& \left.-2^{-3 / 2}\left[k^{1 / 2}-(k-1)^{1 / 2}\right]\right\} \\
& +O\left(n^{-3 / 2}\right) .
\end{aligned}
$$

The principal term in (1.18) was first given by Gronwall [5, 6], and later by Szegö $[14,15]$ with simpler proofs.

An improved version of (1.12) is

$$
\begin{aligned}
L_{n}\left(-\frac{1}{2}, \beta\right)= & \frac{4}{\pi^{2}} \log n+C_{\beta}+E_{\beta} n^{-1}+\frac{1}{\sqrt{\pi}} D_{\beta} n^{-\beta-3 / 2} \\
& +O\left(n^{-2} \log n\right),
\end{aligned}
$$

valid for $-\frac{1}{2}<\beta<\frac{1}{2}$, where $D_{\beta}$ is as given in (1.16) and

$$
E_{\beta}=\frac{4}{\pi^{2}}\left(\frac{\beta+\frac{3}{2}}{2}\right) \text {. }
$$


Finally, we turn to the case $-1<\alpha<-\frac{1}{2}$. Under the additional restrictions $\alpha-\beta>-1$ and $-\frac{1}{2}<\beta<\frac{1}{2}$, we have the following sharpened form of (1.8):

$$
L_{n}(\alpha, \beta)=C_{0}+C_{1} n^{\alpha+1 / 2}+C_{2} n^{\alpha-1 / 2}+C_{3} n^{\alpha-\beta-1}+O\left(n^{-2}\right),
$$

where

$$
C_{0}=\frac{2^{-\alpha}}{\Gamma(\alpha+1)} \int_{0}^{\infty} x^{\alpha}\left|J_{\alpha+1}(x)\right| d x
$$

$$
\begin{gathered}
C_{1}=\frac{4}{\Gamma(\alpha+1) \pi^{3 / 2}} \\
\cdot\left\{\frac{1}{\alpha+1 / 2}\left(\frac{\pi}{2}\right)^{\alpha+1 / 2}\right. \\
\left.\quad+\int_{0}^{\pi / 2}\left[(\sin \theta)^{\alpha-1 / 2}(\cos \theta)^{\beta+1 / 2}-\theta^{\alpha-1 / 2}\right] d \theta\right\} \\
C_{2}=\frac{1}{2}(\alpha+\beta+2)\left(\alpha+\frac{1}{2}\right) C_{1} \\
C_{3}=\frac{1}{\Gamma(\alpha+1)} D_{\beta},
\end{gathered}
$$

$D_{\beta}$ again being the same constant given in (1.16).

Lorch's method essentially consists of replacing the Jacobi polynomial in (1.3) by its asymptotic formula of "Hilb's type" [17, p. 197], and splitting the interval of integration $(O, \pi)$ at the points $j_{\alpha+1, k} / N$, $k=1, \ldots,[N]$, where $N=n+\frac{1}{2}(\alpha+\beta+2)$. Our approach differs from that of Lorch. We first split the interval $(0, \pi)$ at the exact zeros of the Jacobi polynomial and then apply recently obtained uniform asymptotic expansions for the Jacobi polynomials and their zeros [2]. Our method may also be extended to give higher order approximations when desired.

2. Sketch of the procedure. For simplicity of presentation, we restrict our attention to the case $-\frac{1}{2}<\alpha<\frac{1}{2}$ and $-\frac{1}{2}<\beta<\frac{1}{2}$. Let $\theta_{k}$ denote the $k$ th zero of $P_{n}^{(\alpha+1, \beta)}(\cos \theta)$, put $\bar{n}=[n / 2]$, and write

$$
\begin{aligned}
& \frac{\Gamma(\alpha+1) \Gamma(n+\beta+1)}{\Gamma(n+\alpha+\beta+2)} L_{n}(\alpha, \beta) \\
&=\int_{0}^{\theta_{\bar{n}}}+\int_{\theta_{\bar{n}}}^{\pi} \equiv L_{n}^{(1)}(\alpha, \beta)+L_{n}^{(2)}(\alpha, \beta) .
\end{aligned}
$$

We shall first be concerned with the constant $L_{n}^{(1)}(\alpha, \beta)$. The evaluation of $L_{n}^{(2)}(\alpha, \beta)$ proceeds in a similar manner. For convenience, we set 
$\theta_{0} \equiv 0$ and define

$$
I_{k}=\int_{\theta_{k}}^{\theta_{k+1}}\left(\sin \frac{\theta}{2}\right)^{2 \alpha+1}\left(\cos \frac{\theta}{2}\right)^{2 \beta+1} P_{n}^{(\alpha+1, \beta)}(\cos \theta) d \theta .
$$

Since $\cos 0=1$ and

$$
P_{n}^{(\alpha+1, \beta)}(1)=\left(\begin{array}{c}
n+\underset{n}{\alpha}+1 \\
{ }^{(\alpha)}
\end{array}\right)=\frac{\Gamma(n+\alpha+2)}{\Gamma(n+1) \Gamma(\alpha+2)}>0
$$

we have

$$
L_{n}^{(1)}(\alpha, \beta)=\sum_{k=0}^{\bar{n}-1}(-1)^{k} I_{k} .
$$

To evaluate $I_{k}$, we shall use the following results given in [2]; see, in particular, the main theorem, Corollary 2, and the first paragraph in $\S 5$ of that reference.

LEMMA 1. For $\alpha+1>-\frac{1}{2}$ and $\alpha+\beta+1 \geq-1$, we have

$$
\begin{aligned}
& \left(\sin \frac{\theta}{2}\right)^{\alpha+1}\left(\cos \frac{\theta}{2}\right)^{\beta} P_{n}^{(\alpha+1, \beta)}(\cos \theta) \\
& \quad=\frac{\Gamma(n+\alpha+2)}{n !}\left(\frac{\theta}{\sin \theta}\right)^{1 / 2}\left[\sum_{l=0}^{m-1} A_{l}(\theta) \frac{J_{\alpha+l+1}(N \theta)}{N^{\alpha+l+1}}+\sigma_{m}\right],
\end{aligned}
$$

where

$$
N=n+\frac{1}{2}(\alpha+\beta+2)
$$

and

$$
\sigma_{m}=\theta^{m} O\left(N^{-m-\alpha-1}\right),
$$

the O-term being uniform with respect to $\theta \in[0, \pi-\varepsilon], \varepsilon>0$. The coefficients $A_{l}(\theta)$ are analytic functions in $0 \leq \theta \leq \pi-\varepsilon$, and are $O\left(\theta^{l}\right)$ in that interval. In particular, $A_{0}(\theta)=1$ and

$$
A_{1}(\theta)=\left[(\alpha+1)^{2}-\frac{1}{4}\right]\left(\frac{1-\theta \cot \theta}{2 \theta}\right)-\frac{(\alpha+1)^{2}-\beta^{2}}{4} \tan \frac{\theta}{2} .
$$

LeMMA 2. Let $\alpha+1>-\frac{1}{2}, \alpha+\beta+1 \geq-1$ and let $0<\theta_{1}<\theta_{2}<$ $\cdots<\theta_{n}<\pi$ be the zeros of $P_{n}^{(\alpha+1, \beta)}(\cos \theta)$. Then, as $n \rightarrow \infty$,

$$
\begin{aligned}
\theta_{l}= & \frac{j_{\alpha+1, l}}{N} \\
& +\frac{1}{N^{2}}\left\{\left[(\alpha+1)^{2}-\frac{1}{4}\right] \frac{1-t \cot t}{2 t}-\frac{(\alpha+1)^{2}-\beta^{2}}{4} \tan \frac{t}{2}\right\} \\
& +t^{2} O\left(N^{-3}\right),
\end{aligned}
$$


where $j_{\alpha+1, l}$ is the l-th positive zero of the Bessel function $J_{\alpha+1}(x)$ and $t=j_{\alpha+1, l} / N$. The O-term is uniformly bounded for all values of $l=$ $1,2, \ldots,[\gamma n]$, where $\gamma \in(0,1)$ is a constant.

Taking $m=3$ in (2.5) and substituting the resulting expression in (2.2) gives

$$
I_{k}=\frac{\Gamma(n+\alpha+2)}{n ! N^{\alpha+1}}\left[I_{k}^{(1)}+I_{k}^{(2)}+I_{k}^{(3)}+I_{k}^{(4)}\right]
$$

where

$$
I_{k}^{(l+1)}=\frac{1}{N^{l}} \int_{\theta_{k}}^{\theta_{k+1}} A_{l}^{*}(\theta) J_{\alpha+l+1}(N \theta) d \theta, \quad l=0,1,2
$$

and

$$
I_{k}^{(4)}=\frac{1}{N^{3}} O\left\{\int_{\theta_{k}}^{\theta_{k+1}} \theta^{3}\left(\sin \frac{\theta}{2}\right)^{\alpha}\left(\cos \frac{\theta}{2}\right)^{\beta+1}\left(\frac{\theta}{\sin \theta}\right)^{1 / 2} d \theta\right\}
$$

with

$$
A_{l}^{*}(\theta)=\left(\frac{\theta}{2} \cot \frac{\theta}{2}\right)^{1 / 2}\left(\sin \frac{\theta}{2}\right)^{\alpha}\left(\cos \frac{\theta}{2}\right)^{\beta} A_{l}(\theta) .
$$

Note that the implied constant in the $O$-symbol in (2.12) is independent of $k$. It is easily seen that

$$
\sum_{k=0}^{\bar{n}-1}(-1)^{k} I_{k}^{(4)}=O\left(N^{-3}\right)
$$

Using the identity

$$
\frac{d}{d z}\left(z^{-\nu} J_{\nu}(z)\right)=-z^{-\nu} J_{\nu+1}(z),
$$

we have by integration by parts

$$
I_{k}^{(2)}=T_{1}+T_{2},
$$

where

$$
\begin{gathered}
T_{1}=\frac{1}{N^{2}}\left[A_{1}^{*}\left(\theta_{k}\right) J_{\alpha+1}\left(N \theta_{k}\right)-A_{1}^{*}\left(\theta_{k+1}\right) J_{\alpha+1}\left(N \theta_{k+1}\right)\right] \\
T_{2}=\frac{1}{N^{2}} \int_{\theta_{k}}^{\theta_{k+1}}\left[\theta^{\alpha+1} A_{1}^{*}(\theta)\right]^{\prime} \theta^{-\alpha-1} J_{\alpha+1}(N \theta) d \theta .
\end{gathered}
$$

For fixed $\nu$, it is well-known that

(2.19) $j_{\nu, k}=\left(k+\frac{1}{2} \nu-\frac{1}{4}\right) \pi-\frac{4 \nu^{2}-1}{8\left(k+\frac{1}{2} \nu-\frac{1}{4}\right) \pi}+O\left(\frac{1}{k^{3}}\right)$. 
Thus, by Lemma 2, we have

$$
N \theta_{k}=j_{\alpha+1, k}+O\left(k / N^{2}\right),
$$

where the implied constant in the $O$-symbol is independent of $k$ for $k=1, \ldots, \bar{n}$. Note that a combination of (2.6), (2.19) and (2.20) shows that for large $n$, we have $\theta_{\bar{n}}<\pi / 2$. From (2.20), it also follows that

$$
J_{\alpha+1}\left(N \theta_{k}\right)=J_{\alpha+1}\left(j_{\alpha+1, k}\right)+J_{\alpha+1}^{\prime}(\xi) O\left(k / N^{2}\right),
$$

where $\xi$ lies between $N \theta_{k}$ and $j_{\alpha+1, k}$ and hence is $O(k)$. Since $J_{\alpha+1}\left(j_{\alpha+1, k}\right)=0$ and $J_{\alpha+1}^{\prime}(\xi)=O\left(\xi^{-1 / 2}\right)$ as $\xi \rightarrow \infty,(2.21)$ yields

$$
J_{\alpha+1}\left(N \theta_{k}\right)=O\left(k^{1 / 2} / N^{2}\right) .
$$

Observe that $A_{1}^{*}(\theta)=O\left(\theta^{\alpha+1}\right)$ and $\theta_{k}=O(k / N)$. Thus, by coupling (2.17) and (2.22), we obtain

$$
T_{1}=O\left(k^{\alpha+3 / 2} / N^{\alpha+5}\right) .
$$

Furthermore, since $\left[\theta^{\alpha+1} A_{1}^{*}(\theta)\right]^{\prime}=O\left(\theta^{2 \alpha+1}\right)$, it is easily seen that

$$
T_{2}=\frac{1}{N^{\alpha+3}} O\left\{\int_{N \theta_{k}}^{N \theta_{h+1}} y^{\alpha}\left|J_{\alpha+1}(y)\right| d y\right\} .
$$

The integral inside of the curly bracket is clearly a bounded function of $N$ if $k=0$, in view of $(2.20)$. For $k=1, \ldots, \bar{n}-1$, we use (2.19) and the fact $J_{\alpha+1}(y)=O\left(y^{-1 / 2}\right)$ to conclude that this integral is $O\left(k^{\alpha-1 / 2}\right)$. Now we recall the well-known expansions [11, p. 292]

$$
\sum_{k=1}^{n-1} k^{\alpha}-\zeta(-\alpha) \sim \frac{n^{\alpha+1}}{\alpha+1} \sum_{s=0}^{\infty}\left(\begin{array}{c}
\alpha+1 \\
s
\end{array}\right) \frac{B_{s}}{n^{s}} \quad(\alpha \neq-1),
$$

where $\zeta(\mu)$ is the Riemann-Zeta function. A combination of (2.23), (2.24) and (2.25) gives

$$
\sum_{k=0}^{\bar{n}-1}(-1)^{k} I_{k}^{(2)}=O\left(N^{-5 / 2}\right) .
$$

An entirely similar argument leads to

$$
\sum_{k=0}^{\bar{n}-1}(-1)^{k} I_{k}^{(3)}=O\left(N^{-5 / 2}\right) \text {. }
$$

Here we have used the fact that

$$
\bar{n}=\frac{n}{2}\left[1+O\left(\frac{1}{n}\right)\right], \quad \text { as } n \rightarrow \infty .
$$

From (2.4) and (2.10), it now follows that as $n \rightarrow \infty$,

(2.29) $L_{n}^{(1)}(\alpha, \beta)=\frac{\Gamma(n+\alpha+2)}{n ! N^{\alpha+1}}\left[\sum_{k=0}^{\bar{n}-1}(-1)^{k} I_{k}^{(1)}+O\left(N^{-5 / 2}\right)\right]$. 
In the next section, it will be shown that

$$
\begin{aligned}
& \text { (2.30) } \sum_{k=0}^{\bar{n}-1}(-1)^{k} I_{k}^{(1)} \\
& =\frac{1}{2^{\alpha} N^{\alpha+1}}\left\{\int_{0}^{j_{1}} y^{\alpha} J_{\alpha+1}(y) d y+M_{1}(\alpha)\right. \\
& +\sqrt{\frac{2}{\pi}} 2^{(\alpha-\beta-1) / 2} n^{\alpha-1 / 2}+2 S_{n}+2 \alpha R_{n} \\
& \left.+O\left(N^{\alpha-3 / 2}\right)\right\}
\end{aligned}
$$

where

$$
\begin{gathered}
S_{n}=\sum_{k=2}^{\bar{n}-1} g_{\alpha \beta}\left(\theta_{k}\right) M_{k}(\alpha), \\
R_{n}=\sum_{k=1}^{\bar{n}-1}(-1)^{k} \int_{j_{k}}^{j_{k+1}} y^{\alpha-1} J_{\alpha}(y) d y
\end{gathered}
$$

and

$$
g_{\alpha \beta}(\theta)=\left(\frac{\theta}{2} \cot \frac{\theta}{2}\right)^{1 / 2}\left[\frac{\sin \theta / 2}{\theta / 2}\right]^{\alpha}\left(\cos \frac{\theta}{2}\right)^{\beta} .
$$

To proceed further, we need the following two lemmas, whose proofs are given in $\$ 4$.

LEMMA 3. As $n \rightarrow \infty$, the sum in (2.31) has the asymptotic approxima. tion

$$
\begin{gathered}
S_{n}=B_{\alpha}^{(1)}+\frac{\sqrt{2}}{\pi^{3 / 2}} N^{\alpha+1 / 2}\left[\int_{0}^{\pi / 2} g_{\alpha \beta}(\theta) \theta^{\alpha-1 / 2} d \theta\right. \\
-\int_{0}^{j_{1} / N} g_{\alpha \beta}(\theta) \theta^{\alpha-1 / 2} d \theta \\
\left.\quad-\int_{j_{\bar{n}-1} / N}^{\pi / 2} g_{\alpha \beta}(\theta) \theta^{\alpha-1 / 2} d \theta\right] \\
+\frac{n^{\alpha-1 / 2}}{\sqrt{2 \pi}} 2^{(\alpha-\beta-1) / 2}+O\left(n^{\alpha-3 / 2}\right),
\end{gathered}
$$

where $B_{\alpha}^{(1)}$ is a constant given by

$$
B_{\alpha}^{(1)}=\sum_{k=2}^{\infty}\left[M_{k}(\alpha)-\frac{\sqrt{2}}{\pi^{3 / 2}} \int_{j_{k-1}}^{j_{k}} x^{\alpha-1 / 2} d x\right] .
$$


LEMMA 4. The asymptotic behavior of the sum in (2.32) is given by

$$
R_{n}=B_{\alpha}^{(2)}+O\left(n^{\alpha-3 / 2}\right),
$$

where

$$
B_{\alpha}^{(2)}=\sum_{k=1}^{\infty}(-1)^{k} \int_{j_{k}}^{j_{k+1}} x^{\alpha-1} J_{\alpha}(x) d x .
$$

The constants $B_{\alpha}^{(1)}$ and $B_{2}^{(2)}$ are related to the constant $B_{\alpha}$ given in (1.10). The asymptotic approximation of $L_{n}^{(1)}(\alpha, \beta)$ is obtained by inserting (2.34) and (2.36) in (2.30), and combining the resulting expression with (2.29). It is anticipated that the terms involving the last two integrals in (2.34) will combine with similar terms from $L_{n}^{(2)}(\alpha, \beta)$.

Evaluation of $L_{n}^{(2)}(\alpha, \beta)$ proceeds as follows. By definition we have (2.38) $\quad L_{n}^{(2)}(\alpha, \beta)$

$$
=\int_{\theta_{\bar{n}}}^{\pi}\left(\sin \frac{\theta}{2}\right)^{2 \alpha+1}\left(\cos \frac{\theta}{2}\right)^{2 \beta+1}\left|P_{n}^{(\alpha+1, \beta)}(\cos \theta)\right| d \theta,
$$

In the above integral, we replace $\theta$ by $\pi-\theta$. The result is

(2.39) $\quad L_{n}^{(2)}(\alpha, \beta)$

$$
=\int_{0}^{\pi-\theta_{\bar{n}}}\left(\sin \frac{\theta}{2}\right)^{2 \beta+1}\left(\cos \frac{\theta}{2}\right)^{2 \alpha+1}\left|P_{n}^{(\beta, \alpha+1)}(\cos \theta)\right| d \theta,
$$

on account of the identity $[17$, p. 59]

$$
P_{n}^{(\alpha, \beta)}(\cos \theta)=(-1)^{n} P_{n}^{(\beta, \alpha)}(-\cos \theta) .
$$

From (2.40), it also follows that there is a one-to-one relationship between the zeros of $P_{n}^{(\alpha, \beta)}$ and $P_{n}^{(\beta, \alpha)}$. Suppose the zeros of $P_{n}^{(\alpha, \beta)}(\cos \theta)$ are arranged in the order:

$$
0<\theta_{n, 1}^{(\alpha, \beta)}<\theta_{n, 2}^{(\alpha, \beta)}<\cdots<\theta_{n, n}^{(\alpha, \beta)}<\pi .
$$

Since $\theta_{n, 1}^{(\alpha, \beta)}$ is the smallest zero of $P_{n}^{(\alpha, \beta)}(\cos \theta)$, it follows that $\pi-\theta_{n, 1}^{(\alpha, \beta)}$ is the largest zero of $P_{n}^{(\beta, \alpha)}(\cos \theta)$. Thus, $\pi-\theta_{n, 1}^{(\alpha, \beta)}=\theta_{n, n}^{(\beta, \alpha)}$. In general, we have $\pi-\theta_{n, p}^{(\alpha, \beta)}=\theta_{n, n-p+1}^{(\beta, \alpha)}$, or equivalently

$$
\theta_{n, p}^{(\alpha, \beta)}+\theta_{n, n-p+1}^{(\beta, \alpha)}=\pi
$$

for $p=1, \ldots, n$. Since $\theta_{\bar{n}} \equiv \theta_{n, \bar{n}}^{(\alpha+1, \beta)},(2.41)$ gives $\pi-\theta_{\bar{n}}=\theta_{n, n-\bar{n}+1}^{(\beta, \alpha+1)}$. Set $\bar{m}=n-\bar{n}+1$ and $\theta_{\bar{m}} \equiv \theta_{n, \bar{m}}^{(\beta, \alpha+1)}$. Equation (2.39) now becomes

(2.42) $\quad L_{n}^{(2)}(\alpha, \beta)$

$$
=\int_{0}^{\theta_{\bar{m}}}\left(\sin \frac{\theta}{2}\right)^{2 \beta+1}\left(\cos \frac{\theta}{2}\right)^{2 \alpha+1}\left|P_{n}^{(\beta, \alpha+1)}(\cos \theta)\right| d \theta .
$$


Despite the fact that $\theta_{\bar{m}}>\pi / 2$ while $\theta_{n}<\pi / 2$ for large values of $n$, computation of $L_{n}^{(2)}(\alpha, \beta)$ proceeds in the same manner as $L_{n}^{(1)}(\alpha, \beta)$. A brief summary of this calculuation is given in $\$ 5$. Asymptotic expansion (1.14) is obtained by adding the results for $L_{n}^{(1)}(\alpha, \beta)$ and $L_{n}^{(2)}(\alpha, \beta)$ together. This is done in $\S 6$. Since the derivations of expansions (1.19) and (1.21) are similar to that of (1.14), they will not be presented here.

3. Calculation of $I_{k}^{(1)}$. From (2.11) and (2.13) we have

$$
I_{k}^{(1)}=\int_{\theta_{k}}^{\theta_{k+1}} A_{0}^{*}(\theta) J_{\alpha+1}(N \theta) d \theta
$$

where

$$
A_{0}^{*}(\theta)=\left(\frac{\theta}{2} \cot \frac{\theta}{2}\right)^{1 / 2}\left(\sin \frac{\theta}{2}\right)^{\alpha}\left(\cos \frac{\theta}{2}\right)^{\beta} .
$$

As in (2.16), integration by parts gives

$$
I_{k}^{(1)}=F_{1}+F_{2},
$$

for $k \neq 0$, where

$$
F_{1}=\frac{1}{N}\left[A_{0}^{*}\left(\theta_{k}\right) J_{\alpha}\left(N \theta_{k}\right)-A_{0}^{*}\left(\theta_{k+1}\right) J_{\alpha}\left(N \theta_{k+1}\right)\right]
$$

and

$$
F_{2}=\frac{1}{N} \int_{\theta_{k}}^{\theta_{k+1}}\left[A_{0}^{*}(\theta) \theta^{\alpha}\right]^{\prime} \theta^{-\alpha} J_{\alpha}(N \theta) d \theta .
$$

In terms of the function $g_{\alpha \beta}(\theta)$ defined in (2.33) (and also used in [9, (30)]), (3.4) becomes

$$
\begin{aligned}
F_{1}=\frac{1}{2^{\alpha} N^{\alpha+1}}[ & g_{\alpha \beta}\left(\theta_{k}\right)\left(N \theta_{k}\right)^{\alpha} J_{\alpha}\left(N \theta_{k}\right) \\
& \left.-g_{\alpha \beta}\left(\theta_{k+1}\right)\left(N \theta_{k+1}\right)^{\alpha} J_{\alpha}\left(N \theta_{k+1}\right)\right] .
\end{aligned}
$$

By (2.20), Taylor's theorem gives

(3.7) $J_{\alpha}\left(N \theta_{k}\right)=J_{\alpha}\left(j_{\alpha+1, k}\right)+J_{\alpha}^{\prime}\left(j_{\alpha+1, k}\right) O\left(\frac{k}{N^{2}}\right)+J_{\alpha}^{\prime \prime}(\xi) O\left(\frac{k^{2}}{N^{4}}\right)$,

where $\xi$ lies between $N \theta_{k}$ and $j_{\alpha+1, k}$. Since $j_{\alpha+1, k} \sim \pi k$ by (2.19), $\xi=O(k)$ and hence $J_{\alpha}^{\prime \prime}(\xi)=O\left(k^{-1 / 2}\right)$. From the identity $z J_{\alpha}^{\prime}(z)+$ $z J_{\alpha+1}(z)=\alpha J_{\alpha}(z)$, we have

$$
J_{\alpha}^{\prime}\left(j_{\alpha+1, k+1}\right)=\frac{\alpha}{j_{\alpha+1, k+1}} J_{\alpha}\left(j_{\alpha+1, k}\right)=O\left(k^{-3 / 2}\right) .
$$


Therefore, (3.7) becomes

$$
J_{\alpha}\left(N \theta_{k}\right)=J_{\alpha}\left(j_{\alpha+1, k}\right)+O\left(\frac{1}{k^{1 / 2} N^{2}}\right)+O\left(\frac{k^{3 / 2}}{N^{4}}\right) .
$$

Since (2.19) and (2.20) imply

$$
\left(N \theta_{k}\right)^{\alpha}=j_{\alpha+1, k}^{\alpha}+O\left(\frac{k^{\alpha}}{N^{2}}\right)
$$

it follows from (3.6) that

$$
\begin{aligned}
F_{1}= & \frac{(-1)^{k}}{2^{\alpha} N^{\alpha+1}}\left[g_{\alpha \beta}\left(\theta_{k}\right) M_{k}(\alpha)+g_{\alpha \beta}\left(\theta_{k+1}\right) M_{k+1}(\alpha)\right] \\
& +O\left(\frac{k^{\alpha-1 / 2}}{N^{\alpha+3}}\right)
\end{aligned}
$$

where $M_{k}(\alpha)$ is as given in (1.11). In deriving (3.11), we have also used the fact that $g_{\alpha \beta}(\theta)=1+O\left(\theta^{2}\right)$ for $0 \leq \theta \leq \pi / 2$.

We now evaluate $F_{2}$ given in (3.5). From (3.2), we obtain

$$
\left[A_{0}^{*}(\theta) \theta^{\alpha}\right]^{\prime}=\frac{\alpha}{2^{\alpha-1}} \theta^{2 \alpha-1}+O\left(\theta^{2 \alpha+1}\right) .
$$

Inserting (3.12) in (3.5) gives

$$
F_{2}=\frac{\alpha}{2^{\alpha-1} N^{\alpha+1}} Q+R
$$

where

$$
Q=\int_{N \theta_{k}}^{N \theta_{k+1}} y^{\alpha-1} J_{\alpha}(y) d y
$$

and

$$
R=\frac{1}{N^{\alpha+3}} O\left\{\int_{N \theta_{k}}^{N \theta_{k+1}} y^{\alpha+1}\left|J_{\alpha}(y)\right| d y\right\} .
$$

In view of (2.19), (2.20) and the behavior of the Bessel function, it is easily seen that the integral in (3.15) is equal to

$$
\left|\int_{j_{k}}^{j_{k+1}} y^{\alpha+1} J_{\alpha}(y) d y\right|+O\left(\frac{k^{\alpha+3 / 2}}{N^{2}}\right) .
$$

Since $x^{\alpha+1} J_{\alpha}(x)=\left[x^{\alpha+1} J_{\alpha+1}(x)\right]^{\prime}$, the last integral is zero. Thus

$$
R=O\left(k^{\alpha+3 / 2} / N^{\alpha+5}\right) \text {. }
$$

By the same argument, we also have

$$
Q=\int_{j_{k}}^{j_{k+1}} y^{\alpha-1} J_{\alpha}(y) d y+O\left(\frac{k^{\alpha-1 / 2}}{N^{2}}\right) .
$$


Now substitute (3.16) and (3.17) in (3.13), and add the resulting equation to (3.11). From (3.3) it follows that the sum is

$$
\begin{aligned}
I_{k}^{(1)}= & \frac{(-1)^{k}}{2^{\alpha} N^{\alpha+1}}\left[g_{\alpha \beta}\left(\theta_{k}\right) M_{k}(\alpha)+g_{\alpha \beta}\left(\theta_{k+1}\right) M_{k+1}(\alpha)\right] \\
& +\frac{\alpha}{2^{\alpha-1} N^{\alpha+1}} \int_{j_{k}}^{j_{k+1}} y^{\alpha-1} J_{\alpha}(y) d y+O\left(\frac{k^{\alpha-1 / 2}}{N^{\alpha+3}}\right)
\end{aligned}
$$

for $k \neq 0$.

Note that (3.2) gives

$$
A_{0}^{*}(\theta)=\left(\frac{\theta}{2}\right)^{\alpha}\left[1+O\left(\theta^{2}\right)\right]
$$

Inserting this in (3.1), we get

$$
\text { (3.20) } I_{0}^{(1)}=\frac{1}{2^{\alpha}} \int_{0}^{\theta_{1}} \theta^{\alpha} J_{\alpha+1}(N \theta) d \theta+O\left\{\int_{0}^{\theta_{1}} \theta^{\alpha+2} J_{\alpha+1}(N \theta) d \theta\right\} \text {. }
$$

Since (2.20) implies $\theta_{1}=O\left(N^{-1}\right)$, the second integral in (3.20) is $O\left(N^{-\alpha-3}\right)$. By a similar argument, it can easily be shown that the first integral is equal to

$$
\frac{1}{N^{\alpha+1}} \int_{0}^{j_{1}} y^{\alpha} J_{\alpha+1}(y) d y+O\left(\frac{1}{N^{\alpha+3}}\right) .
$$

Thus we have

$$
I_{0}^{(1)}=\frac{1}{2^{\alpha} N^{\alpha+1}} \int_{0}^{j_{1}} y^{\alpha} J_{\alpha+1}(y) d y+O\left(\frac{1}{N^{\alpha+3}}\right) .
$$

From (2.25), (3.18) and (3.21), it now follows that

$$
\begin{aligned}
\sum_{k=0}^{\bar{n}-1}(-1)^{k} I_{k}^{(1)} & \frac{1}{2^{\alpha} N^{\alpha+1}} \sum_{k=1}^{\bar{n}-1}\left[g_{\alpha \beta}\left(\theta_{k}\right) M_{k}(\alpha)+g_{\alpha \beta}\left(\theta_{k+1}\right) M_{k+1}(\alpha)\right] \\
& +\frac{1}{2^{\alpha} N^{\alpha+1}} \int_{0}^{j_{1}} y^{\alpha} J_{\alpha+1}(y) d y+\frac{\alpha}{2^{\alpha-1} N^{\alpha+1}} R_{n} \\
& +O\left(\frac{1}{N^{\alpha+3}}\right)+O\left(N^{-5 / 2}\right)
\end{aligned}
$$

where $R_{n}$ is given in (2.32). The sum on the right-hand side of (3.22) can be written as

$$
\begin{aligned}
& \sum_{k=1}^{\bar{n}-1}\left[g_{\alpha \beta}\left(\theta_{k}\right) M_{k}(\alpha)+g_{\alpha \beta}\left(\theta_{k+1}\right) M_{k+1}(\alpha)\right] \\
& =g_{\alpha \beta}\left(\theta_{1}\right) M_{1}(\alpha)+g_{\alpha \beta}\left(\theta_{\bar{n}}\right) M_{\bar{n}}(\alpha)+2 S_{n}
\end{aligned}
$$


with $S_{n}$ being given in (2.31). Since $g_{\alpha \beta}(\theta)=1+O\left(\theta^{2}\right)$ and $\theta_{1}=j_{1} / N$ $+O\left(N^{-3}\right)$, we obtain

$$
g_{\alpha \beta}\left(\theta_{1}\right) M_{1}(\alpha)=M_{1}(\alpha)+O\left(N^{-2}\right) .
$$

Inserting (2.19) in the asymptotic expansion of $J_{\alpha}(x)$ gives

(3.25) $M_{k}(\alpha)=\sqrt{\frac{2}{\pi}}\left[j_{\alpha+1, k}^{\alpha-1 / 2}-\frac{1}{4}\left(\alpha+\frac{1}{2}\right)\left(\alpha+\frac{3}{2}\right) j_{\alpha+1, k}^{\alpha-5 / 2}+O\left(j_{\alpha+1, k}^{\alpha-7 / 2}\right)\right]$; cf. (1.11). This in particular gives

$$
M_{\bar{n}}(\alpha)=\left(\frac{\pi}{2}\right)^{\alpha-1} n^{\alpha-1 / 2}+O\left(n^{\alpha-3 / 2}\right) .
$$

Since $g_{\alpha \beta}(\theta)$ is analytic in $[0, \pi)$ (see (2.33)), we may expand it at $\theta=\pi / 2$. Thus,

$$
g_{\alpha \beta}\left(\theta_{\bar{n}}\right)=g_{\alpha \beta}\left(\frac{\pi}{2}+O\left(n^{-1}\right)\right)=\left(\frac{\pi}{4}\right)^{1 / 2-\alpha} 2^{-(\alpha+\beta) / 2}+O\left(n^{-1}\right),
$$

and consequently

$$
g_{\alpha \beta}\left(\theta_{\bar{n}}\right) M_{\bar{n}}(\alpha)=\sqrt{\frac{2}{\pi}} 2^{(\alpha-\beta-1) / 2} n^{\alpha-1 / 2}+O\left(n^{\alpha-3 / 2}\right) .
$$

A combination of (3.22), (3.23), (3.24) and (3.28) yields the desired result (2.30).

4. Proofs of Lemmas 3 and 4. We first give the proof of Lemma 4, which is considerably simpler than that of Lemma 3. Using the identity $\left[x^{\alpha+1} J_{\alpha+1}(x)\right]^{\prime}=x^{\alpha+1} J_{\alpha}(x)$, we have by integration by parts

$$
\int_{j_{k}}^{j_{k+1}} x^{\alpha-1} J_{\alpha}(x) d x=2 \int_{j_{k}}^{j_{k+1}} x^{\alpha-2} J_{\alpha+1}(x) d x \quad(k \neq 0),
$$

the integrated term vanishing since $j_{k}$ and $j_{k+1}$ are zeros of $J_{\alpha+1}(x)$. In view of the asymptotic behavior of $J_{\alpha}(x)$, the last integral is $O\left(k^{\alpha-5 / 2}\right)$. Thus

$$
\sum_{k=\bar{n}}^{\infty}(-1)^{k} \int_{j_{k}}^{j_{k+1}} x^{\alpha-1} J_{\alpha}(x) d x=O\left(n^{\alpha-3 / 2}\right) .
$$

This establishes Lemma 4.

Now we begin the proof of Lemma 3. By using (2.19) and (3.25), it can be shown that for $k \neq 1$,

$$
\begin{aligned}
M_{k}(\alpha)= & \frac{2^{1 / 2}}{\pi^{3 / 2}} \int_{j_{k-1}}^{j_{k}} x^{\alpha-1 / 2} d x+\frac{\alpha-1 / 2}{\sqrt{2 \pi}} \int_{j_{k-1}}^{j_{k}} x^{\alpha-3 / 2} d x \\
& +O\left(k^{\alpha-5 / 2}\right) .
\end{aligned}
$$


Thus

$$
\begin{aligned}
\sum_{k=\bar{n}}^{\infty}\left[M_{k}(\alpha)\right. & -\frac{2^{1 / 2}}{\pi^{3 / 2}} \int_{j_{k-1}}^{j_{k}} x^{\alpha-1 / 2} d x \\
& \left.-\frac{\alpha-1 / 2}{\sqrt{2 \pi}} \int_{j_{k-1}}^{j_{k}} x^{\alpha-3 / 2} d x\right]=O\left(n^{\alpha-3 / 2}\right) .
\end{aligned}
$$

Set

$$
\begin{aligned}
S_{n, 1}=\sum_{k=2}^{\infty}\left[M_{k}(\alpha)\right. & -\frac{2^{1 / 2}}{\pi^{3 / 2}} \int_{j_{k-1}}^{j_{k}} x^{\alpha-1 / 2} d x \\
& \left.-\frac{\alpha-1 / 2}{\sqrt{2 \pi}} \int_{j_{k-1}}^{j_{k}} x^{\alpha-3 / 2} d x\right]
\end{aligned}
$$

$$
S_{n, 2}=\frac{2^{1 / 2}}{\pi^{3 / 2}} \sum_{k=2}^{\bar{n}-1} \int_{j_{k-1}}^{j_{k}} g_{\alpha \beta}\left(\theta_{k}\right) x^{\alpha-1 / 2} d x
$$

and

$$
S_{n, 3}=\frac{\alpha-1 / 2}{(2 \pi)^{1 / 2}} \sum_{k=2}^{\bar{n}-1} \int_{j_{k-1}}^{j_{k}} g_{\alpha \beta}\left(\theta_{k}\right) x^{\alpha-3 / 2} d x .
$$

Since $g_{\alpha \beta}\left(\theta_{k}\right)=1+O\left(k^{2} / N^{2}\right)$, we can now express $S_{n}$ as

$$
S_{n}=S_{n, 1}+S_{n, 2}+S_{n, 3}+O\left(n^{\alpha-3 / 2}\right) \text {. }
$$

Note that the series $S_{n, 1}$ converges absolutely in view of (4.1), and is a constant independent of $n$.

(A) Evaluation of $S_{n, 2}$. Make the change of variable $x=\theta N$ in (4.4), and write

$$
g_{\alpha \beta}\left(\theta_{k}\right)=g_{\alpha \beta}(\theta)+g_{\alpha \beta}^{\prime}(\theta)\left(\theta_{k}-\theta\right)+\frac{1}{2} g_{\alpha \beta}^{\prime \prime}(\xi)\left(\theta_{k}-\theta\right)^{2},
$$

where $\xi$ is between $\theta_{k}$ and $\theta$. Since $\theta \in\left[j_{k-1} / N, j_{k} / N\right]$, by (2.20) we have $\theta_{k}-\theta=O(1 / N)$. Furthermore, since $g_{\alpha \beta}(\theta)$ is analytic in $[0, \pi / 2]$, $g_{\alpha \beta}^{\prime \prime}(\xi)$ is bounded. Thus the remainder term $\frac{1}{2} g_{\alpha \beta}^{\prime \prime}(\xi)\left(\theta_{k}-\theta\right)^{2}$ is $O\left(n^{-2}\right)$, and contributes to $S_{n, 2}$ a term of order

$$
O\left(n^{-2} \sum_{k=2}^{\bar{n}-1} \int_{j_{k-1}}^{j_{k}} x^{\alpha-1 / 2} d x\right)=O\left(n^{-2} \sum_{k=2}^{\bar{n}-1} k^{\alpha-1 / 2}\right)=O\left(n^{\alpha-3 / 2}\right) .
$$

Inserting (4.7) in (4.4) then leads to

$$
S_{n, 2}=\frac{2^{1 / 2}}{\pi^{3 / 2}}\left[S_{n, 2}^{(1)}+S_{n, 2}^{(2)}+S_{n, 2}^{(3)}\right]+O\left(n^{\alpha-3 / 2}\right)
$$

where

$$
S_{n, 2}^{(1)}=N^{\alpha+1 / 2} \sum_{k=2}^{\bar{n}-1} \int_{j_{k-1} / N}^{j_{k}} g_{\alpha \beta}(\theta) \theta^{\alpha-1 / 2} d \theta
$$




$$
S_{n, 2}^{(2)}=N^{\alpha+1 / 2} \sum_{n=2}^{n-1} \theta_{k} \int_{j_{k-1} / N}^{j_{k} / N} g_{\alpha \beta}^{\prime}(\theta) \theta^{\alpha-1 / 2} d \theta
$$

and

$$
S_{n, 2}^{(3)}=-N^{\alpha+1 / 2} \sum_{k=2}^{\bar{n}-1} \int_{j_{k-1} / N}^{j_{k} / N} g_{\alpha \beta}^{\prime}(\theta) \theta^{\alpha+1 / 2} d \theta .
$$

Clearly, $S_{n, 2}^{(1)}$ can be written as

$$
S_{n, 2}^{(1)}=N^{\alpha+1 / 2}\left[\int_{0}^{\pi / 2}-\int_{0}^{j_{1} / N}-\int_{j_{\bar{n}-1 / N}}^{\pi / 2}\right] g_{\alpha \beta}(\theta) \theta^{\alpha-1 / 2} d \theta
$$

which is exactly the second term on the right-hand side of (2.34) (except for the constant factor $\left.\sqrt{2} / \pi^{3 / 2}\right)$.

In (4.11), we let $g_{\alpha \beta}^{\prime}(\theta) \theta^{\alpha-1 / 2}=f(\theta)$. With an appropriate change of variable, each integral there can be written as

$$
\begin{aligned}
\int_{j_{k-1} / N}^{j_{k} / N} \theta f(\theta) d \theta & \\
= & \frac{1}{2 N}\left(j_{k}+j_{k-1}\right) \int_{j_{k-1} / N}^{j_{k} / N} f(\theta) d \theta \\
& +\int_{0}^{\left(j_{k}-j_{k-1}\right) / 2 N} \theta\left[f\left\{\frac{1}{2 N}\left(j_{k}+j_{k-1}\right)+\theta\right\}\right. \\
& \left.\quad-f\left\{\frac{1}{2 N}\left(j_{k}+j_{k-1}\right)-\theta\right\}\right] d \theta .
\end{aligned}
$$

By the mean value theorem the second integral on the right is equal to

$$
\int_{0}^{\left(j_{k}-j_{k-1}\right) / 2 N} 2 \theta^{2} f^{\prime}\left(\xi_{\theta}\right) d \theta
$$

for some $\xi_{\theta}$ satisfying

$$
\frac{1}{2 N}\left(j_{k}+j_{k-1}\right)-\theta<\xi_{\theta}<\frac{1}{2 N}\left(j_{k}+j_{k-1}\right)+\theta .
$$

Since $0 \leq \theta \leq\left(j_{k}-j_{k-1}\right) / 2 N$ in (4.14), we have $j_{k-1} / N \leq \xi_{\theta} \leq j_{k} / N$ so that, by (2.19), $\xi_{\theta}=O(k / N)$. From (2.19) it also follows that $\left(j_{k}-j_{k-1}\right) / 2 N=O(1 / N)$. Note that the implied constants in the last two $O$-terms are independent of $k$. Since $f^{\prime}(\theta)=O\left(\theta^{\alpha-1 / 2}\right)$, a combination of the above results shows that the integral in (4.14) is $O\left(k^{\alpha-1 / 2} / N^{\alpha+5 / 2}\right)$. By using this estimate and (4.13), the addition of (4.10) and (4.11) yields

(4.15) $S_{n, 2}^{(2)}+S_{n, 2}^{(3)}$

$$
\begin{aligned}
= & N^{\alpha+1 / 2} \sum_{k=2}^{\bar{n}-1}\left\{\left[\theta_{k}-\frac{1}{2 N}\left(j_{k}+j_{k-1}\right)\right] \int_{j_{k-1} / N}^{j_{k} / N} g_{\alpha \beta}^{\prime}(\theta) \theta^{\alpha-1 / 2} d \theta\right\} \\
& +O\left(N^{\alpha-3 / 2}\right),
\end{aligned}
$$


where we have also made use of (2.25). From (2.19) and (2.20), it now follows that for $k \geq 1$,

$$
\theta_{k}-\frac{1}{2 N}\left(j_{k}+j_{k-1}\right)=\frac{\pi}{2 N}+O\left(\frac{1}{k^{2} N}\right)+O\left(\frac{k}{N^{3}}\right) .
$$

Since $g_{\alpha \beta}(\theta)=1+O\left(\theta^{2}\right)$ for $0 \leq \theta \leq \pi / 2$, each integral under the summation sign in (4.15) is $O\left(k^{\alpha+1 / 2} / N^{\alpha+3 / 2}\right)$, where again the implied constant in the last $O$-term is independent of $k$. Since $-\frac{1}{2}<\alpha<\frac{1}{2}$, a combination of (4.15) with (4.16), (2.25) and the last estimate yields

$$
\begin{aligned}
S_{n, 2}^{(2)}+S_{n, 2}^{(3)}= & \frac{\pi}{2} N^{\alpha-1 / 2} \sum_{k=2}^{\bar{n}-1} \int_{j_{k-1} / N}^{j_{k} / N} g_{\alpha \beta}^{\prime}(\theta) \theta^{\alpha-1 / 2} d \theta \\
& +O\left(N^{\alpha-3 / 2}\right) .
\end{aligned}
$$

The sum in (4.17) may now be written as

$$
\begin{aligned}
\int_{0}^{\pi / 2} g_{\alpha \beta}^{\prime}(\theta) \theta^{\alpha-1 / 2} d \theta & -\int_{0}^{j_{1} / N} g_{\alpha \beta}^{\prime}(\theta) \theta^{\alpha-1 / 2} d \theta \\
& -\int_{j_{\bar{n}-1} / N}^{\pi / 2} g_{\alpha \beta}^{\prime}(\theta) \theta^{\alpha-1 / 2} d \theta .
\end{aligned}
$$

The second integral in (4.18) is $O\left(N^{-\alpha-3 / 2}\right)$ and, since $j_{\bar{n}-1} / N=\pi / 2+$ $O\left(N^{-1}\right)$, the third integral is $O\left(N^{-1}\right)$. Coupling (4.17) and (4.18) results in

$$
S_{n, 2}^{(2)}+S_{n, 2}^{(3)}=\frac{\pi}{2} N^{\alpha-1 / 2} \int_{0}^{\pi / 2} g_{\alpha \beta}^{\prime}(\theta) \theta^{\alpha-1 / 2} d \theta+O\left(N^{\alpha-3 / 2}\right) .
$$

Finally, using (4.8), the addition of (4.12) and (4.19) gives

$$
\text { (4.20) } \begin{aligned}
S_{n, 2}= & \frac{2^{1 / 2}}{\pi^{3 / 2}} N^{\alpha+1 / 2}\left[\int_{0}^{\pi / 2}-\int_{0}^{j_{1} / N}-\int_{j_{\bar{n}-1 / N}}^{\pi / 2}\right] g_{\alpha \beta}(\theta) \theta^{\alpha-1 / 2} d \theta \\
& +\frac{1}{\sqrt{2 \pi}} N^{\alpha-1 / 2} \int_{0}^{\pi / 2} g_{\alpha \beta}^{\prime}(\theta) \theta^{\alpha-1 / 2} d \theta+O\left(n^{\alpha-3 / 2}\right) .
\end{aligned}
$$

(B) Evaluation of $S_{n, 3}$. The analysis here parallels that given for $S_{n, 2}$, and is in fact simpler. We first make the change of variable $x=\theta N$ in (4.5), and then substitute (4.7) in (4.5). The result is

$$
S_{n, 3}=\frac{\alpha-1 / 2}{(2 \pi)^{1 / 2}} N^{\alpha-1 / 2}\left[S_{n, 3}^{(1)}+S_{n, 3}^{(2)}\right]+O\left(n^{-2}\right),
$$

where

$$
S_{n, 3}^{(1)}=\int_{j_{1} / N}^{j_{\bar{n}-1} / N} g_{\alpha \beta}(\theta) \theta^{\alpha-3 / 2} d \theta
$$

and

$$
S_{n, 3}^{(2)}=\sum_{k=2}^{\bar{n}-1} \int_{j_{k-1} / N}^{j_{k} / N} g_{\alpha \beta}^{\prime}(\theta)\left(\theta_{k}-\theta\right) \theta^{\alpha-3 / 2} d \theta ;
$$


cf. (4.8). To the integral in (4.22), we apply integration by parts. By using (3.27) and the fact that $g_{\alpha \beta}(\theta)=1+O\left(\theta^{2}\right)$ as $\theta \rightarrow 0^{+}$, it can be shown that the integrated term is equal to

$$
\frac{1}{\alpha-1 / 2}\left[2^{(\alpha-\beta-1) / 2}-\left(j_{1} / N\right)^{\alpha-1 / 2}+O\left(n^{-1}\right)\right] .
$$

The other term can be replaced by

$$
\frac{1}{\alpha-1 / 2} \int_{0}^{\pi / 2} g_{\alpha \beta}^{\prime}(\theta) \theta^{\alpha-1 / 2} d \theta+O\left(n^{-1}\right),
$$

as was done in the case of $S_{n, 2}$, cf. (4.18). Thus

$$
\begin{aligned}
S_{n, 3}^{(1)}= & \frac{1}{\alpha-1 / 2}\left[2^{(\alpha-\beta-1) / 2}-\left(j_{1} / N\right)^{\alpha-1 / 2}\right. \\
& \left.\quad-\int_{0}^{\pi / 2} g_{\alpha \beta}^{\prime}(\theta) \theta^{\alpha-1 / 2} d \theta\right] \\
& +O\left(n^{-1}\right) .
\end{aligned}
$$

Since $g_{\alpha \beta}^{\prime}=O(\theta)$ and $\theta_{k}-\theta=O\left(n^{-1}\right)$ for $j_{k-1} / N \leq \theta \leq j_{k} / N$, the $(k-1)$ th integral in (4.23) is $O\left(k^{\alpha-1 / 2} / N^{\alpha+3 / 2}\right)$. This implies

$$
S_{n, 3}^{(2)}=O\left(N^{-1}\right) \text {. }
$$

The sum of $S_{n, 3}^{(1)}$ and $S_{n, 3}^{(2)}$ gives, using (4.21),

$$
\begin{aligned}
S_{n, 3}= & -\frac{j_{1}^{\alpha-1 / 2}}{\sqrt{2 \pi}}+\frac{N^{\alpha-1 / 2}}{\sqrt{2 \pi}}\left[2^{(\alpha-\beta-1) / 2}-\int_{0}^{\pi / 2} g_{\alpha \beta}^{\prime}(\theta) \theta^{\alpha-1 / 2} d \theta\right] \\
& +O\left(n^{\alpha-3 / 2}\right),
\end{aligned}
$$

thus completing the evaluation of $S_{n, 3}$.

Observing that the sum $S_{n, 1}$ in (4.3) can be written as

$$
S_{n, 1}=B_{\alpha}^{(1)}+\frac{j_{1}^{\alpha-1 / 2}}{\sqrt{2 \pi}}
$$

where $B_{\alpha}^{(1)}$ is given in (2.35), the result in (2.34) now follows immediately from (4.6), (4.20) and (4.26). This proves Lemma 3.

5. Evaluation of $L_{n}^{(2)}(\alpha, \beta)$. In what follows we shall use the same notation as we did in the evaluation of $L_{n}^{(1)}(\alpha, \beta)$. There should be no confusion resulting from this, when care is taken to distinguish the zeros of $P_{n}^{(\alpha+1, \beta)}(\cos \theta)$ and $P_{n}^{(\beta, \alpha+1)}(\cos \theta)$. Thus we again let $\theta_{0} \equiv 0, \theta_{k}$ denote the $k$ th positive zero of $P_{n}^{(\beta, \alpha+1)}(\cos \theta)$, and

$$
I_{k}=\int_{\theta_{k}}^{\theta_{k+1}}\left(\sin \frac{\theta}{2}\right)^{2 \beta+1}\left(\cos \frac{\theta}{2}\right)^{2 \alpha+1} P_{n}^{(\beta, \alpha+1)}(\cos \theta) d \theta
$$


Since $\cos 0=1$ and

$$
P_{n}^{(\beta, \alpha+1)}(1)=\frac{\Gamma(n+\beta+1)}{\Gamma(n+1) \Gamma(\beta+1)}>0,
$$

we have from (2.42)

$$
L_{n}^{(2)}(\alpha, \beta)=\sum_{k=0}^{\bar{m}-1}(-1)^{k} I_{k} .
$$

As before, we now use the asymptotic expansion of $P_{n}^{(\beta, \alpha+1)}(\cos \theta)$ for large $n$. The result corresponding to (2.29) is

$$
L_{n}^{(2)}(\alpha, \beta)=\frac{\Gamma(n+\beta+1)}{n ! N^{\beta}}\left[\sum_{k=0}^{\bar{m}-1}(-1)^{k} I_{k}^{(1)}+O\left(n^{-5 / 2}\right)\right],
$$

where

$$
\begin{aligned}
& \sum_{k=0}^{\bar{m}-1}(-1)^{k} I_{k}^{(1)} \\
& =\frac{1}{2^{\beta+1} N^{\beta+2}}\left\{\int_{0}^{j_{\beta, 1}} y^{\beta+1} J_{\beta}(y) d y+\hat{M}_{1}(\beta)\right. \\
& \left.\quad+\sqrt{\frac{2}{\pi}} 2^{(\beta-\alpha+1) / 2} n^{\beta+1 / 2}+2 \hat{S}_{n}+O\left(n^{\beta-1 / 2}\right)\right\}, \\
& \hat{S}_{n}=\sum_{k=2}^{\bar{m}-1} \hat{g}_{\alpha \beta}\left(\theta_{k}\right) \hat{M}_{k}(\beta),
\end{aligned}
$$

$\hat{M}_{k}(\beta)$ being as given in (1.17), and

$$
\hat{g}_{\alpha \beta}(\theta)=\left(\frac{2}{\theta} \tan \frac{\theta}{2}\right)^{1 / 2}\left(\frac{2}{\theta} \sin \frac{\theta}{2}\right)^{\beta}\left(\cos \frac{\theta}{2}\right)^{\alpha} .
$$

Note that $\hat{R}_{n}$, the contribution to $L_{n}^{(2)}(\alpha, \beta)$ corresponding to $R_{n}$ in (2.32), is absent in (5.5), because

$$
\int_{j_{\beta, k}}^{j_{\beta, k+1}} y^{\beta} J_{\beta-1}(y) d y=\int_{j_{\beta, k}}^{j_{\beta, k+1}}\left[y^{\beta} J_{\beta}(y)\right]^{\prime} d y=0 .
$$

An analogue of Lemma 3 is the following approximation:

$$
\begin{aligned}
\hat{S}_{n}= & \frac{\sqrt{2}}{\pi^{3 / 2}} N^{\beta+3 / 2}\left(\int_{0}^{\pi / 2}-\int_{0}^{j_{\beta, 1} / N}-\int_{j_{\beta, \bar{m}-1} / N}^{\pi / 2}\right) \hat{g}_{\alpha \beta}(\theta) \theta^{\beta+1 / 2} d \theta \\
& +\sqrt{\frac{2}{\pi}} 2^{(\beta-\alpha-1) / 2} N^{\beta+1 / 2}+2^{\beta} D_{\beta}-\hat{M}_{1}(\beta) \\
& +\frac{2^{1 / 2}}{\pi^{3 / 2}\left(\beta+\frac{3}{2}\right)} j_{\beta, 1}^{\beta+3 / 2}+O\left(N^{\beta-1 / 2}\right),
\end{aligned}
$$


where $D_{\beta}$ is given in (1.16). Note that the sum in $D_{\beta}$ starts with $k=1$, whereas the sum in $B_{\alpha}^{(1)}$, the corresponding constant in $S_{n}$ given in (2.35), starts with $k=2$. Since $\hat{g}_{\alpha \beta}(\theta)=1+O\left(\theta^{2}\right)$, the term involving the second integral on the right of (5.8) cancels with the third from the last term in the same equation. Thus

$$
\begin{aligned}
\hat{S}_{n}= & \frac{\sqrt{2}}{\pi^{3 / 2}} N^{\beta+3 / 2}\left(\int_{0}^{\pi / 2}-\int_{j_{\beta, \bar{m}-1} / N}^{\pi / 2}\right) \hat{g}_{\alpha \beta}(\theta) \theta^{\beta+1 / 2} d \theta \\
& +\sqrt{\frac{2}{\pi}} 2^{(\beta-\alpha-1) / 2} N^{\beta+1 / 2}+2^{\beta} D_{\beta}-\hat{M}_{1}(\beta) \\
& +O\left(N^{\beta-1 / 2}\right) .
\end{aligned}
$$

Now we insert (5.9) in (5.5), and observe that

$$
\int_{0}^{j_{\beta, 1}} x^{\beta+1} J_{\beta}(x) d x=\hat{M}_{1}(\beta)
$$

which follows from (1.17) and the identity $\left[x^{\beta+1} J_{\beta+1}(x)\right]^{\prime}=x^{\beta+1} J_{\beta}(x)$. The resulting expression from (5.5) coupled with (5.4) gives

$$
\begin{aligned}
& \frac{\Gamma(n+\alpha+\beta+2)}{\Gamma(\alpha+1) \Gamma(n+\beta+1)} L_{n}^{(2)}(\alpha, \beta) \\
& =\frac{2^{-1-\beta}}{\Gamma(\alpha+1)}\left[n^{\alpha-\beta-1}+\frac{1}{2}(\alpha+\beta+2)(\alpha-\beta-1) n^{\alpha-\beta-2}\right] \\
& \quad \cdot\left\{2^{\beta+1} D_{\beta}+2 \sqrt{\frac{2}{\pi}} 2^{(\beta-\alpha+1) / 2} n^{\beta+1 / 2}\right. \\
& \quad+\frac{2^{3 / 2}}{\pi^{3 / 2}} N^{\beta+3 / 2}\left(\int_{0}^{\pi / 2}-\int_{j_{\beta, \bar{m}} / 2}^{\pi / 2}\right) \\
& \left.\quad \cdot 2^{\beta+1 / 2}\left(\sin \frac{\theta}{2}\right)^{\beta+1 / 2}\left(\cos \frac{\theta}{2}\right)^{\alpha-1 / 2} d \theta\right\} \\
& +O\left(n^{\alpha-3 / 2}\right),
\end{aligned}
$$

where use has been made of (5.7) and the asymptotic expansion

$$
\begin{aligned}
& \frac{2^{-1-\beta} \Gamma(n+\alpha+\beta+2)}{\Gamma(\alpha+1) \Gamma(n+1) N^{2 \beta+2}} \\
= & \frac{2^{-1-\beta}}{\Gamma(\alpha+1)} n^{\alpha-\beta-1}\left[1+\frac{1}{2 n}(\alpha+\beta+2)(\alpha-\beta-1)+O\left(n^{-2}\right)\right] .
\end{aligned}
$$


6. The sum of $L_{n}^{(1)}(\alpha, \beta)$ and $L_{n}^{(2)}(\alpha, \beta)$. From (2.29), (2.30) and the fact that

$$
\frac{\Gamma(n+\alpha+\beta+2)}{\Gamma(n+\beta+1)} \frac{\Gamma(n+\alpha+2)}{n ! N^{2 \alpha+2}}=1+O\left(n^{-2}\right),
$$

we have

$$
\begin{aligned}
& \frac{\Gamma(n+\alpha+\beta+2)}{\Gamma(\alpha+1) \Gamma(n+\beta+1)} L_{n}^{(1)}(\alpha, \beta) \\
& =\frac{1}{2^{\alpha} \Gamma(\alpha+1)}\left\{\int_{0}^{j_{1}} y^{\alpha} J_{\alpha+1}(y) d y+M_{1}(\alpha)\right. \\
& \left.\quad+\sqrt{\frac{2}{\pi}} 2^{(\alpha-\beta-1) / 2} n^{\alpha-1 / 2}+2 S_{n}+2 \alpha R_{n}\right\} \\
& \quad+O\left(N^{\alpha-3 / 2}\right) .
\end{aligned}
$$

Since $g_{\alpha \beta}(\theta)=1+O\left(\theta^{2}\right)$, the second integral on the right-hand side of (2.34) can be written as

(6.3) $\int_{0}^{j_{1} / N} g_{\alpha \beta}(\theta) \theta^{\alpha-1 / 2} d \theta=\frac{1}{N^{\alpha+1 / 2}} \int_{0}^{j_{1}} x^{\alpha-1 / 2} d x+O\left(N^{-\alpha-5 / 2}\right)$.

Now write (2.35) as

$$
\begin{aligned}
B_{\alpha}^{(1)}= & \sum_{k=1}^{\infty}\left[M_{k}(\alpha)-\frac{\sqrt{2}}{\pi^{3 / 2}} \int_{j_{k-1}}^{j_{k}} x^{\alpha-1 / 2} d x\right] \\
& -M_{1}(\alpha)+\frac{\sqrt{2}}{\pi^{3 / 2}} \int_{0}^{j_{1}} x^{\alpha-1 / 2} d x,
\end{aligned}
$$

where we have used the fact that $j_{0}=0$. Combining (6.1), (2.34), (2.36), (6.2) and (6.3) gives

$$
\begin{aligned}
& \frac{\Gamma(n+\alpha+\beta+2)}{\Gamma(\alpha+1) \Gamma(n+\beta+1)} L_{n}^{(1)}(\alpha, \beta) \\
& =B_{\alpha}+\frac{1}{2^{\alpha} \Gamma(\alpha+1)} \\
& \quad \cdot \frac{2^{\alpha+1}}{\pi^{3 / 2}} N^{\alpha+1 / 2} \int_{0}^{\pi / 2}\left(\sin \frac{\theta}{2}\right)^{\alpha-1 / 2}\left(\cos \frac{\theta}{2}\right)^{\beta+1 / 2} d \theta \\
& \quad+\frac{2^{3 / 2}}{\pi^{3 / 2}} 2^{(\alpha-\beta-1) / 2} n^{\alpha-1 / 2} \\
& \left.\quad-\frac{2^{\alpha+1}}{\pi^{3 / 2}} N^{\alpha+1 / 2} \int_{j_{n}-1 / N}^{\pi / 2}\left(\sin \frac{\theta}{2}\right)^{\alpha-1 / 2}\left(\cos \frac{\theta}{2}\right)^{\beta+1 / 2} d \theta\right\} \\
& +O\left(n^{\alpha-3 / 2}\right),
\end{aligned}
$$

where $B_{\alpha}$ is given in (1.10) and we have used (2.33) and (2.6). 
We now note that

$$
\begin{gathered}
{\left[n^{\alpha-\beta-1}+\frac{1}{2}(\alpha+\beta+2)(\alpha-\beta-1) n^{\alpha-\beta-2}\right] N^{\beta+3 / 2}} \\
=n^{\alpha+1 / 2}+C_{\alpha \beta} n^{\alpha-1 / 2}+O\left(n^{\alpha-3 / 2}\right),
\end{gathered}
$$

where $C_{\alpha \beta}$ is given in (1.15). Upon adding (6.5) and (5.11) and using (6.6), we obtain

$$
\begin{aligned}
L_{n}(\alpha, \beta)= & I_{1}^{*}+I_{2}^{*}+I_{3}^{*}+B_{\alpha}+\frac{1}{\Gamma(\alpha+1)} D_{\beta} \\
& +O\left(n^{\alpha-3 / 2}\right),
\end{aligned}
$$

where $B_{\alpha}$ and $D_{\beta}$ are given in (1.10) and (1.16) respectively and where

$$
\begin{aligned}
I_{1}^{*}= & \frac{2}{\pi^{3 / 2} \Gamma(\alpha+1)}\left(n^{\alpha+1 / 2}+C_{\alpha \beta} n^{\alpha-1 / 2}\right) \\
& \cdot\left\{\int_{0}^{\pi / 2}\left(\sin \frac{\theta}{2}\right)^{\alpha-1 / 2}\left(\cos \frac{\theta}{2}\right)^{\beta+1 / 2} d \theta\right. \\
& \left.+\int_{0}^{\pi / 2}\left(\sin \frac{\theta}{2}\right)^{\beta+1 / 2}\left(\cos \frac{\theta}{2}\right)^{\alpha-1 / 2} d \theta\right\} \\
& +O\left(n^{\alpha-3 / 2}\right),
\end{aligned}
$$

(6.9) $I_{2}^{*}=-\frac{2 n^{\alpha+1 / 2}}{\Gamma(\alpha+1)}\left[\int_{j_{\bar{n}-1} / N}^{\pi / 2}\left(\sin \frac{\theta}{2}\right)^{\alpha-1 / 2}\left(\cos \frac{\theta}{2}\right)^{\beta+1 / 2} d \theta\right.$

$$
\left.+\int_{j_{\beta, \bar{m}-1} / N}^{\pi / 2}\left(\sin \frac{\theta}{2}\right)^{\beta+1 / 2}\left(\cos \frac{\theta}{2}\right)^{\alpha-1 / 2} d \theta\right]
$$

and

$$
I_{3}^{*}=\frac{4}{\Gamma(\alpha+1)} \sqrt{\frac{2}{\pi}} 2^{-(\alpha+\beta+1) / 2} n^{\alpha-1 / 2} .
$$

By letting $\theta=\pi-\phi$ in the second integral in (6.8), the two integrals there can be combined into the single integral

$$
\int_{0}^{\pi}\left(\sin \frac{\theta}{2}\right)^{\alpha-1 / 2}\left(\cos \frac{\theta}{2}\right)^{\beta+1 / 2} d \theta=\frac{\Gamma\left(\frac{1}{2} \alpha+\frac{1}{4}\right) \Gamma\left(\frac{1}{2} \beta+\frac{3}{4}\right)}{\Gamma\left(\frac{1}{2}(\alpha+\beta)+1\right)} .
$$

Using the above result in (6.8) yields

$$
I_{1}^{*}=A_{\alpha \beta} n^{\alpha+1 / 2}+C_{\alpha \beta} A_{\alpha \beta} n^{\alpha-1 / 2}+O\left(n^{\alpha-3 / 2}\right),
$$

where $A_{\alpha \beta}$ is given in (1.6). Making the change of variable $\theta=\pi-\phi$ in the second integral in (6.9), the two integrals there can also be combined 
into the single integral

$$
\int_{j_{\bar{n}-1} / N}^{\pi-j_{\beta, \bar{m}-1} / N}\left(\sin \frac{\theta}{2}\right)^{\alpha-1 / 2}\left(\cos \frac{\theta}{2}\right)^{\beta+1 / 2} d \theta .
$$

Note that by (2.20) and (2.41), we have

$$
\pi-\frac{j_{\beta, \bar{m}-1}}{N}=\theta_{\bar{n}+1}+O\left(n^{-2}\right), \quad \frac{j_{\bar{n}-1}}{N}=\theta_{\bar{n}-1}+O\left(n^{-2}\right) .
$$

From (2.19) and (2.20), it also follows that

$$
\theta_{\bar{n}+1}=\frac{\pi}{2}+O\left(n^{-1}\right), \quad \theta_{\bar{n}-1}=\frac{\pi}{2}+O\left(n^{-1}\right),
$$

and

$$
\theta_{\bar{n}+1}-\theta_{\bar{n}-1}=\frac{2 \pi}{n}+O\left(n^{-2}\right) .
$$

Expanding the integrand in (6.13) about $\pi / 2$ and using the above results, the integral in (6.13) can be shown to be

$$
\frac{2 \pi}{n} 2^{-(\alpha+\beta) / 2}+O\left(n^{-2}\right) .
$$

This result coupled with (6.9) gives

(6.14) $I_{2}^{*}=-\frac{4}{\Gamma(\alpha+1)} \sqrt{\frac{2}{\pi}} 2^{-(\alpha+\beta+1) / 2} n^{\alpha-1 / 2}+O\left(n^{\alpha-3 / 2}\right)$.

A combination of (6.7), (6.10), (6.12) and (6.14) yields our final result (1.14).

To conclude this paper, we consider the particular case of Laplace series given in (1.18). The constant term and the $O\left(n^{-1}\right)$ term in (1.18) have a somewhat different appearance from those obtained by putting $\alpha=\beta=0$ in (1.14). The transition to the form in (1.18) is made by writing the second infinite series in (1.10) as

$$
\begin{aligned}
\lim _{n \rightarrow \infty} & {\left[M_{1}(0)+\cdots+M_{n}(0)-\frac{\sqrt{2}}{\pi^{3 / 2}} \int_{0}^{j_{1, n}} x^{-1 / 2} d x\right] } \\
& =\lim _{n \rightarrow \infty}\left[M_{1}(0)+\cdots+M_{n}(0)-\frac{2^{3 / 2}}{\pi} n^{1 / 2}\right] \\
& =\lim _{n \rightarrow \infty} \sum_{k=1}^{n}\left\{M_{k}(0)-\frac{2^{3 / 2}}{\pi}\left(k^{1 / 2}-[k-1]^{1 / 2}\right)\right\},
\end{aligned}
$$


since from (2.19), $\left(j_{1, n}\right)^{1 / 2}=(n \pi)^{1 / 2}+O\left(n^{-1 / 2}\right)$. Now using the fact that, for $\alpha=0$,

$$
\begin{aligned}
\int_{0}^{j_{1}} x^{\alpha} J_{\alpha+1}(x) d x & =\int_{0}^{j_{1,1}} J_{1}(x) d x \\
& =J_{0}(0)-J_{0}\left(j_{1,1}\right)=1+M_{1}(0),
\end{aligned}
$$

the result for the constant term in (1.18) follows. Similarly in (1.16) we write

$$
\begin{aligned}
D_{0}=\lim _{n \rightarrow \infty}\left[\hat{M}_{1}(0)+\cdots+\hat{M}_{n}(0)\right. & -\frac{\sqrt{2}}{3 / 2} \int_{0}^{j_{0, n}} x^{1 / 2} d x \\
& \left.-\frac{1}{2^{3 / 2} \pi^{1 / 2}} \int_{0}^{j_{0, n}} x^{-1 / 2} d x\right] \\
=\lim _{n \rightarrow \infty} \sum_{k=1}^{n}\left\{\hat{M}_{k}(0)-\frac{2^{3 / 2}}{3}\left(k^{3 / 2}-[k-1]^{3 / 2}\right)\right. & \left.-2^{-3 / 2}\left(k^{1 / 2}-[k-1]^{1 / 2}\right)\right\},
\end{aligned}
$$

where we have used $\left(j_{0, n}\right)^{3 / 2}=\pi^{3 / 2}\left(n^{3 / 2}-\frac{3}{8} n^{1 / 2}\right)+O\left(n^{-1 / 2}\right)$. Grouping these results together yields the form of $L_{n}(0,0)$ given in (1.18).

Acknowledgment. We would like to thank Professor Lee Lorch for posing the problem to us as well as providing some historical background.

\section{REFERENCES}

[1] L. Fejer, Lebesguesche Konstanten und divergente Fourierreihen, J. Reine Angew. Math., 138 (1910), 22-53.

[2] C. L. Frenzen and R. Wong, A uniform asymptotic expansion of the Jacobi polynomials with error bounds, Canad. J. Math., to appear.

[3] _ On the asymptotic behavior of the Lebesgue constants for Jacobi series, C. R. Math. Acad. Sci. Canada, 6 (1984), 267-271.

[4] T. H. Gronwall, Über die Lebesgueschen Konstanten bei den Fourierschen Reihen, Math. Ann., 72 (1912), 244-261.

[5] _ Über die Laplacesche Reihe, Math. Ann., 74 (1913), 213-270.

[6] $\quad$ On the degree of convergence of Laplace series, Trans. Amer. Math. Soc., 15 (1914), 1-30.

[7] G. H. Hardy, Note on Lebesgue's constants in the theory of Fourier series, J. London Math. Soc., 17 (1942), 4-13.

[8] L. Lorch, The Lebesgue constants for Jacobi series, I, Proc. Amer. Math. Soc., 10 (1959), 756-761.

[9] The Lebesgue constants for Jacobi series, II, Amer. J. Math., 81 (1959), 875-888.

[10] , private communication. 
[11] F. W. J. Olver, Asymptotics and Special Functions, Academic Press, New York, 1974.

[12] H. Rau, Über die Lebesgueschen Konstanten der Reihenentwicklungen nach Jacobischen Polynomen, J. Reine Angew. Math., 161 (1929), 237-254.

[13] G. Szegö, Über die Lebesgueschen Konstanten bei den Fourierschen Reihen, in The Collected Papers of Gabor Szegö, Vol. 1, pp. 309-313, R. Askey, ed., Birkhauser, Cambridge, MA, 1982.

[14] _ Über einige asymptotische Entwicklungen der Legendreschen Funktionen, Proc. London Math. Soc., (2), 36 (1932), 427-450.

[15] _ Über eine von Herrn S. Berstein herrührende Abschätzung der Legendreschen Polynome, Math. Ann., 108 (1933), 360-369.

[16] _ Asymptotische Entwicklungen der Jacobischen Polynome, Schr. der König. Gelehr. Gesell. Naturwiss. K1., 10 (1933), 33-112.

[17] , Orthogonal Polynomials, Colloquium Publications, Vol. 23, 3rd ed., Amer. Math. Soc., Providence, RI 1967.

[18] G. N. Watson, The constants of Landau and Lebesgue, Quart. J. Math., 1 (1930), 310-318.

[19] A. Zygmund, Trigonometric Series, Warsaw, 1935.

Received September 4, 1984 and in revised form May 31, 1985. The second author is an I. W. Killam Research Fellow, 1982-1984. The work of this author was also partially supported by N.S.E.R.C. Grant No. A7359.

UNIVERSITY OF BRITISH COLUMBIA,

VANCOUVER, B.C., V6T 1Y4, CANada

AND

UNIVERSITY OF MANITOBA

WinNipeg, Manitoba R3T 2N2, CANADA 



\section{PACIFIC JOURNAL OF MATHEMATICS EDITORS}

\author{
V. S. VARAdarajan (Managing Editor) \\ University of California \\ Los Angeles, CA 90024 \\ Hebert Clemens \\ University of Utah \\ Salt Lake City, UT 84112 \\ Charles R. DePrima \\ California Institute of Technology \\ Pasadena, CA 91125
}

R. FINN

Stanford University

Stanford, CA 94305

HeRManN FLASChKa

University of Arizona

Tucson, AZ 85721

RAMESH A. GANGOlli

University of Washington

Seattle, WA 98195

ROBION KIRBY

University of California

Berkeley, CA 94720

\author{
C. C. MOORE \\ University of California \\ Berkeley, CA 94720 \\ H. SAMELSON \\ Stanford University \\ Stanford, CA 94305 \\ HAROLD STARK \\ University of California, San Diego \\ La Jolla, CA 92093
}

\section{ASSOCIATE EDITORS}

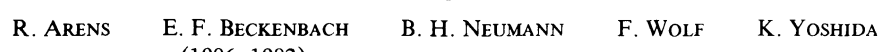

(1906-1982)

\section{SUPPORTING INSTITUTIONS}

\begin{abstract}
UNIVERSITY OF ARIZONA
UNIVERSITY OF BRITISH COLUMBIA

CALIFORNIA INSTITUTE OF TECHNOLOGY

UNIVERSITY OF CALIFORNIA

MONTANA STATE UNIVERSITY

UNIVERSITY OF NEVADA, RENO

NEW MEXICO STATE UNIVERSITY

OREGON STATE UNIVERSITY
\end{abstract}

\author{
UNIVERSITY OF OREGON \\ UNIVERSITY OF SOUTHERN CALIFORNIA \\ STANFORD UNIVERSITY \\ UNIVERSITY OF HAWAII \\ UNIVERSITY OF TOKYO \\ UNIVERSITY OF UTAH \\ WASHINGTON STATE UNIVERSITY \\ UNIVERSITY OF WASHINGTON
}

The Supporting Institutions listed above contribute to the cost of publication of this Journal, but they are not owners or publishers and have no responsibility for its content or policies.

Mathematical papers intended for publication in the Pacific Journal of Mathematics should be in typed form or offset-reproduced (not dittoed), double spaced with large margins. Please do not use built up fractions in the text of the manuscript. However, you may use them in the displayed equations. Underline Greek letters in red, German in green, and script in blue. The first paragraph must be capable of being used separately as a synopsis of the entire paper. In particular it should contain no bibliographic references. Please propose a heading for the odd numbered pages of less than 35 characters. Manuscripts, in triplicate, may be sent to any one of the editors. Please classify according to the scheme of Math. Reviews, Index to Vol. 39. Supply name and address of author to whom proofs should be sent. All other communications should be addressed to the managing editor, or Elaine Barth, University of California, Los Angeles, California 90024.

There are page-charges associated with articles appearing in the Pacific Journal of Mathematics. These charges are expected to be paid by the author's University, Government Agency or Company. If the author or authors do not have access to such Institutional support these charges are waived. Single authors will receive 50 free reprints; joint authors will receive a total of 100 free reprints. Additional copies may be obtained at cost in multiples of 50 .

The Pacific Journal of Mathematics is issued monthly as of January 1966. Regular subscription rate: $\$ 190.00$ a year (5 Vols., 10 issues). Special rate: $\$ 95.00$ a year to individual members of supporting institutions.

Subscriptions, orders for numbers issued in the last three calendar years, and changes of address should be sent to Pacific Journal of Mathematics, P.O. Box 969, Carmel Valley, CA 93924, U.S.A. Old back numbers obtainable from Kraus Periodicals Co., Route 100, Millwood, NY 10546.

The Pacific Journal of Mathematics at P.O. Box 969, Carmel Valley, CA 93924 (ISSN 0030-8730) publishes 5 volumes per year. Application to mail at Second-class postage rates is pending at Carmel Valley, California, and additional mailing offices. Postmaster: Send address changes to Pacific Journal of Mathematics, P.O. Box 969, Carmel Valley, CA 93924.

PUBLISHED BY PACIFIC JOURNAL OF MATHEMATICS, A NON-PROFIT CORPORATION

Copyright $\odot 1986$ by Pacific Journal of Mathematics 


\section{Pacific Journal of Mathematics}

\section{Vol. 122, No. 2 \\ February, 1986}

Gideon Amit and David Chillag, On a question of Feit concerning character values of finite solvable groups ......................257

Constantin Gelu Apostol and Frank Larkin Gilfeather, Isomorphisms modulo the compact operators of nest algebras ................263

Parviz Azimi and James Neil Hagler, Examples of hereditarily $l^{1}$ Banach

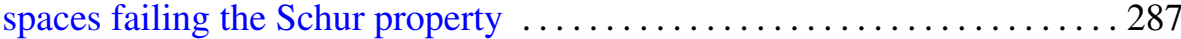

Brian Evan Blank, Boundary behavior of limits of discrete series representations of real rank one semisimple groups . . . . . . . . . . 299

Jeffrey Carroll, Some undecidability results for lattices in recursion theory

Gerald Howard Cliff and Alfred Rheinhold Weiss, Crossed product and

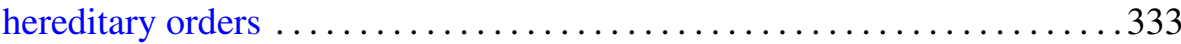

Ralph Cohen, Realizing transfer maps for ramified coverings . . . . . . . . 347

Ronald James Evans, Hermite character sums . .................. 357

C. L. Frenzen and Roderick Sue-Chuen Wong, Asymptotic expansions of the Lebesgue constants for Jacobi series . . . . . . . . . . . . . . . . 391

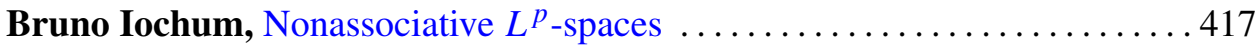

John McDonald, Unimodular approximation in function algebras ....... 435

John Robert Quine, Jr., Ramification and unintegrated value distribution . . 441

Marc Raphael, Commutants of quasisimilar subnormal operators ........ 449

Parameswaran Sankaran and Peter Zvengrowski, On stable

parallelizability of flag manifolds

Helga Schirmer, A relative Nielsen number

Barry Simon, Schrödinger semigroups on the scale of Sobolev spaces . . . . . 475

Viakalathur Shankar Sunder, Stochastic integration in Fock space

Jan de Vries, A note on the $G$-space version of Glicksberg's theorem 